\title{
Aprendizaje con simulación virtual. Una aplicación a la nivelación topográfica Learning with virtual simulation. An application to the topographic leveling
}

\author{
José Manuel Benito Oterino, Marina Martínez Peña, Rosa M. Chueca Castedo \\ josemanuel.benito@upm.es, marina.martinez@upm.es, r.chueca@upm.es \\ Departamento de Ingeniería topográfica y Cartografía \\ ETSI Topografía, Geodesia y Cartografía \\ Universidad Politécnica de Madrid \\ Madrid, España
}

\begin{abstract}
Resumen- La realidad virtual se está revelando como una potente herramienta educativa para generar todo tipo de simuladores, muy valorados tanto por los alumnos, que complementan así la docencia presencial, como por otros usuarios que encuentran en ellos un recurso motivador que facilita su autoaprendizaje. En este trabajo se plantea un laboratorio virtual, particularizado para el caso de la nivelación topográfica, y se describen los módulos que comprende, destacando su potencial como eficaz herramienta para el aprendizaje. Se presentan tres prácticas de nivelación geométrica que constituyen la primera fase del laboratorio virtual de observaciones topográficas TOPLAB de la UPM. Las observaciones topográficas forman parte de los planes de estudio de múltiples grados en ingeniería que desarrollan prácticas de campo, supeditadas a las inclemencias meteorológicas, con instrumental específico muy costoso. Estas prácticas virtuales salvan los contratiempos mencionados además de proporcionar una eficaz retroalimentación al verificar los datos que el alumno registra durante la observación, así como los cálculos que realiza para obtener los resultados oportunos en cada práctica. Este proyecto se enmarca en la línea de producción de recursos didácticos disponibles en red, en abierto, que es objetivo del grupo de Innovación Educativa INNGEO desde 2005.
\end{abstract}

Palabras clave: Simulación virtual, Laboratorio virtual, Observaciones topográficas, Aula invertida, Autoaprendizaje.

Abstract- Virtual reality is revealing itself as a powerful educational tool to generate all kinds of simulators, highly valued both by students who complement face-to-face teaching and by other users who find in them a motivating resource that facilitates their selflearning. In this work, a virtual laboratory is proposed, particularized for the case of topographic leveling, and describes the modules it comprises, highlighting its potential as an effective tool for learning. Three practices of geometric leveling are presented, which constitute the first phase of TOPLAB, topographic measurements virtual lab. The topographic observations are part of the curricula of multiple degrees in engineering that develop field practices, subject to meteorological inclemencies, with very expensive specific instruments. These virtual practices save these setbacks as well as provide effective feedback by verifying the student's data during observation as well as the calculations he makes to obtain the timely results in each practice. This project is part of the production line of didactic resources available in open network that is an objective of the INNGEO Educational Innovation group since 2005.

Keywords: Virtual simulation, Virtual lab, Surveying measurements, Flipped classroom, Self-learning.

\section{INTRODUCCIÓN}

Los mundos virtuales 3D han abierto nuevas posibilidades para la enseñanza y el aprendizaje por medio de la simulación de espacios y experiencias (Márquez, 2010). La fuerte carga visual de estos mundos y el hecho de que los usuarios puedan manejar su propia representación virtual, o avatar, a través del espacio tridimensional, proporcionan a los usuarios una experiencia distinta a los espacios de aprendizaje tradicionales, no sólo físicos, sino también e-learning. Los mundos virtuales pueden acoger también la enseñanza de cuestiones técnicas como por ejemplo la utilización de instrumental y la aplicación de metodologías para la determinación experimental de ciertas magnitudes, tal es el caso de las denominadas “observaciones topográficas”.

La cultura de la simulación, introducida en los ordenadores por Macintosh en 1984, a través de espacios (escritorio) y objetos (carpetas, disquetes, papelera) reales, para representar información y trabajar de un modo más fácil e intuitivo, no ha hecho más que amplificarse.

La relación con los objetos simulados en la pantalla del ordenador es cada vez más parecida a la mantenida con los objetos de la vida real; y lo mismo ocurre con las personas, con los distintos iconos, textos, fotografías y avatares que los representan en la pantalla. No se tiene el más mínimo sentido de irrealidad. La pantalla del ordenador es actualmente un espacio para la interacción, el diálogo, la exploración y, sin duda, para su aplicación a la enseñanza y el aprendizaje

Consciente de la necesidad de introducir actualizaciones adaptadas a la nuevas tecnologías en el proceso enseñanzaaprendizaje, el Grupo de Innovación Educativa INNGEO de la Universidad politécnica de Madrid (Grupo de Innovación Educativa INNGEO, 2005) produce desde 1995 recursos didácticos disponibles en red, abiertos tanto para los alumnos de Ingeniería Geomática cuanto para otros usuarios.

Siguiendo la línea marcada en 2010 con la producción de 2 series de vídeos didácticos sobre observaciones topográficas (Benito Oterino, Martínez Peña, \& Chueca Castedo, 2013), y su publicación en Canal_UPM Youtube (432.000 accesos a junio 2017), y con el fin, no solo, de dar mayor protagonismo al alumno a través de su trabajo, “aprender haciendo”, sino también, convencido del potencial de la simulación, se ha 
concebido ahora un laboratorio virtual de observaciones topográficas en el que se reproducen prácticas topográficas con su instrumental específico.

En este paso adelante, en el que se trabaja el "hacer", además de la profundización en la fase de fijación del aprendizaje, se facilita al alumno la práctica continua, ya que puede emular en el mundo virtual las observaciones topográficas, tanto antes como después de la clase presencial.

Este laboratorio virtual de observaciones topográficas de la UPM, TOPLAB, se revela además como un recurso didáctico potenciador del modelo pedagógico “Aula invertida”, dado que el alumno puede iniciarse en las observaciones topográficas en el mundo virtual para después abordar las prácticas en el campo real con el soporte del profesor.

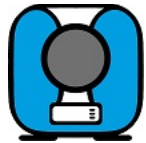

\section{TPAB \\ Laboratorio virtual de observaciones topográficas}

Fig. 1 Logos del laboratorio virtual TOPLAB

En la primera fase del laboratorio, puesta en servicio al final del curso académico 2016-17, se han implementado las tres prácticas que corresponden a las metodologías básicas de la nivelación geométrica con nivel o equialtímetro (Arranz Justel \& Soler García, 2015).

TOPLAB, con la utilización de réplicas de instrumental topográfico y accesorios, es un Laboratorio virtual 3D que no solo permite la realización de la observación topográfica (Fig. 2), sino que incorpora también la toma de datos, los cálculos topográficos elementales consiguientes y la verificación inmediata de los mismos, ofreciendo al alumno una eficaz retroalimentación. Próximamente se incorporará también la segunda fase, consistente en la serie de prácticas correspondientes a la utilización de estaciones totales.

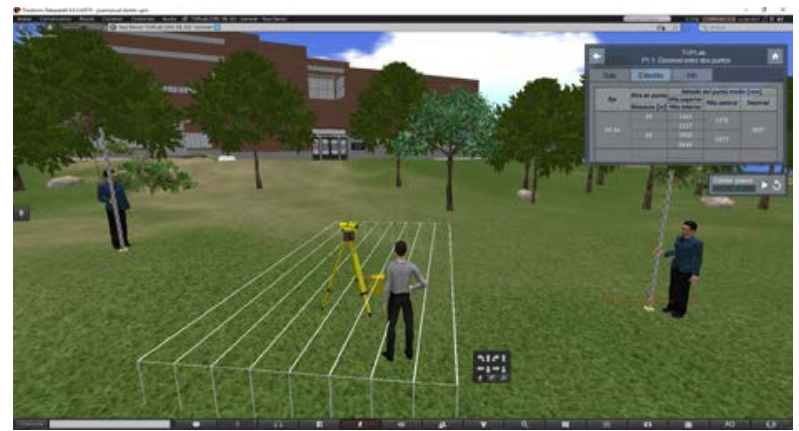

Fig. 2 Campo virtual. Observación del desnivel entre dos puntos por el método de punto medio

TOPLAB constituye un complemento docente que permite a los usuarios adquirir competencias prácticas que por limitaciones de tiempo, climatología o coste del instrumental no pueden desarrollarse presencialmente en campo. Además, permite reforzar en cualquier momento conceptos adquiridos por los estudiantes durante la ejecución de prácticas de campo presenciales, profundizando en aspectos como la utilización de niveles (equialtímetros) o la captura de datos y su procesado, sin límite de tiempo, en cualquier lugar con acceso a internet.

Con el objetivo general de contribuir también a la producción de prácticas virtuales como recurso didáctico, se desarrollan asimismo en este trabajo las diferentes fases en que se ha estructurado la realización del proyecto TOPLAB:
Planificación inicial, creación y modelado de instrumental y accesorios virtuales, adaptación al mundo virtual de las operaciones topográficas que se realizan en el mundo real, revisión de las versiones intermedias y, finalmente, definición de criterios para valorar la bondad de las observaciones virtuales y la correcta ejecución de los cálculos realizados por los alumnos.

\section{CONTEXTO}

\section{A. Necesidad de su realización}

Las observaciones topográficas, integradas en la ingeniería Geomática, forman parte de las enseñanzas de múltiples ramas de la ingeniería por ser la Geomática una ciencia aplicada muy transversal.

La evolución del instrumental topográfico ha supuesto una simplificación en el manejo y utilización de los equipos ya que cuentan con dispositivos electrónicos que facilitan la puesta en estación y la captura y registro de los datos. Aunque no cabe duda de que siempre será conveniente que el alumno realice prácticas reales de observación, la simulación propuesta facilitará y hará más eficaz el trabajo de campo en el mundo real. Además, actualmente, los alumnos están familiarizados con la realidad virtual a través de la utilización de videojuegos, por lo que los laboratorios virtuales se están revelando para ellos como un recurso didáctico muy atractivo y motivador, en este caso para el estudio de la Topografía.

El laboratorio virtual TOPLAB, alojado en la plataforma 3dlabs.upm creada por el GATE ${ }^{1}$ ( GATE UPM, 2017), está desarrollado en el software de código abierto OpenSimulator, que permite crear mundos virtuales a los que se accede mediante el uso de un visor (en este caso, Firestorm). Al entrar en la plataforma, el avatar aparece en una región central de bienvenida, constituida por edificios centrales, un punto de encuentro virtual y una región o isla por laboratorio en la que realizará las prácticas virtuales; además podrá desenvolverse e interaccionar con otros usuarios en salas virtuales de reuniones, salas de video y lugares de esparcimiento.

Los objetos 3D que se muestran en los laboratorios han sido diseñados mediante 3DS Max o bien mediante el uso de primitivas dentro del entorno. Para añadir funcionalidad a los objetos 3D dentro del entorno de OpenSimulator, se utiliza el lenguaje de scripting LSL (Linden Scripting Lenguage).

\section{B. Objetivos}

Las prácticas de nivelación geométrica que ofrece este laboratorio virtual, forman parte de las prácticas de campo básicas que incluyen los planes de estudios en diferentes niveles de enseñanza, desde formación profesional hasta estudios universitarios.

El proyecto TOPLAB nació dirigido, en principio, a profesores y alumnos de la UPM para las asignaturas de Geomática y Topografía en los Grados impartidos en seis escuelas de ingeniería, (ETSI en Topografía Geodesia y Cartografía; de Minas y Energía; de Caminos, Canales y Puertos; de Edificación; Agronómica, Alimentaria y de Biosistemas; de Montes, Forestal y del Medio Natural).

${ }^{1}$ Gabinete de Tele-educación de la Universidad Politécnica de Madrid. Premio Computerworld a la innovación en educación 2016 por el proyecto de Laboratorios virtuales UPM

http://www.upm.es/?id=07977dca6b647510VgnVCM10000009c7648a_ \& \&refmt=articulo\&fmt=detail

Octubre 4-6, 2017, Zaragoza, ESPAÑA 
También está dirigido a profesores y alumnos de otras universidades que imparten enseñanzas relacionadas con las observaciones topográficas, así como de Institutos de Enseñanza Secundaria que imparten Ciclos formativos de grado superior que incluyen la materia “Topografía”.

$\mathrm{Al}$ ofrecer desde la Universidad el laboratorio en abierto, el destinatario final alcanza a cualquier usuario nacional e internacional interesado en el área de la Geomática (en particular en las observaciones topográficas) que disponga de acceso a Internet, como ya ocurre con usuarios de otros recursos didácticos implementados por INNGEO y ofrecidos también en abierto.

\section{DESCRIPCIÓN}

A. Estructura modular de un mundo virtual para el aprendizaje: Aplicación al caso del laboratorio para nivelación geométrica

Las simulaciones permiten abordar situaciones del mundo real desde una perspectiva particular en la que se pueden, por ejemplo, simplificar procesos, adaptar situaciones o fomentar conductas, en favor de organizar el aprendizaje para que resulte más eficaz. Así, la experiencia en un laboratorio virtual en el que se puede entrenar a los alumnos (Ruiz Gutierrez, 2013), supone una adaptación a la experiencia real.

En los diferentes módulos que se pueden considerar en cualquier laboratorio virtual se manifestará dicha adaptación, tal es el caso del laboratorio de observaciones topográficas TOPLAB en el que puede destacarse:

- Módulo terreno

- Módulo instrumental

- Módulo de medición-registro y cálculo

Módulo terreno. La simulación para el aprendizaje tiene sus orígenes en el entrenamiento profesional de pilotos y en los años setenta y ochenta cobró un fuerte impulso con el desarrollo de la tecnología de imágenes 3D interactivas por ordenador que permitió la simulación de las características del paisaje que ve normalmente un piloto y la posibilidad de interactuar con ellas (Márquez, 2010). En TOPLAB se realizan las observaciones topográficas sobre la recreación virtual del campo de prácticas de la Escuela Técnica Superior de Ingenieros en Topografía, Geodesia y Cartografía que cuenta con un Marco de Referencia que incluye más de 200 referencias con coordenadas.

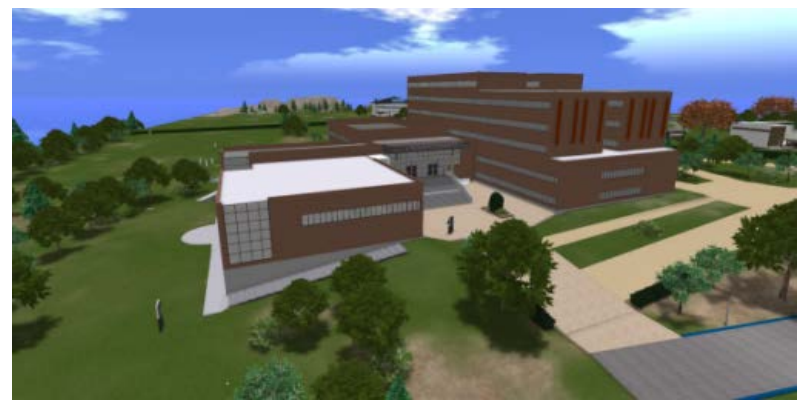

Fig. 3 Campo de prácticas virtual de la ETSITGC UPM

Módulo instrumental. En los laboratorios virtuales, la simulación supone el uso de instrumental que se habrá modelado de tal manera que permita al alumno realizar las mediciones utilizando el ordenador (Ruiz Gutierrez, 2013).
Para las prácticas de nivelación geométrica se han modelado niveles ópticos y electrónicos simplificando algunas funcionalidades de los instrumentos actuales y adaptando los modelos a las necesidades de TOPLAB, para hacerlo más didáctico y accesible a los usuarios en sus primeros contactos con las observaciones topográficas. También se han modelado miras con graduación convencional y con código de barras, además de otros accesorios.

Módulo de medición-registro y cálculo. La simulación en el mundo virtual de los procesos de trabajo real, conlleva la adaptación de los mismos, en general, simplificando el formalismo y rigor con que se utiliza el instrumental en laboratorio. En las prácticas de nivelación, las operaciones topográficas emuladas son:

- Elección de instrumental y emplazamientos correspondientes al objetivo de cada observación.

- Observación propiamente dicha, actuando sobre los elementos de estacionamiento, visado y puntería.

- Captura de datos y su volcado, ordenadamente, sobre los oportunos ficheros de observaciones.

Las simulaciones hacen que los estudiantes sean responsables de su propio aprendizaje y su motivación consiste en la consecución de metas y objetivos. Un usuario que se sienta delante de un ordenador y comienza a conducir una simulación, irá a través de un círculo de aprendizaje: reflexionar sobre el caso, elegir la estrategia, tomar decisiones y observar las consecuencias de esas decisiones (Paniagua, 2006). En TOPLAB, tras la captura y registro de los datos, el alumno (usuario) realizará las comprobaciones de campo y los cálculos oportunos y completará con sus resultados los ficheros de observaciones; en la fase de verificación y retroalimentación, TOPLAB le facilitará sobre sus propios ficheros de observaciones:

- La bondad de las comprobaciones de campo y la coherencia de sus observaciones (gracias a la métrica incorporada y oculta para el usuario).

- La revisión de los cálculos topográficos correspondientes a cada observación.

Las prácticas implementadas, en orden creciente de dificultad, son las que recogen la metodología básica de la nivelación geométrica. Abarcan desde la presentación del método en el supuesto más sencillo, hasta la observación completa de un itinerario de nivelación. Cada práctica puede realizarse independientemente, seleccionándola del menú mostrado al entrar en TOPLAB (Fig. 4), las dos primeras con nivel óptico y la tercera con nivel electrónico ( GATE UPM, 2017).

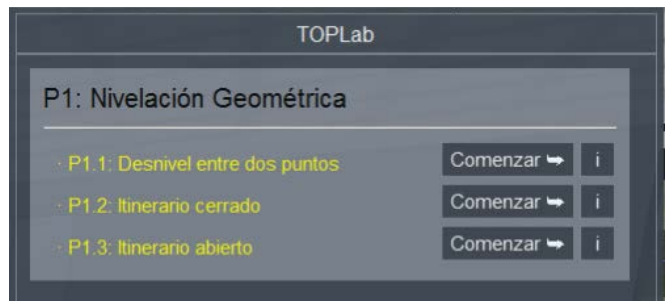

Fig. 4 Menú de Prácticas de nivelación geométrica

TOPLAB dispone de instrumental y referencias señalizadas en campo suficientes para que más de 30 alumnos/usuarios puedan realizar prácticas virtuales simultáneamente. 
B. Desarrollo de las prácticas de nivelación geométrica implementadas

Una vez que en el mundo virtual el avatar haya acudido al laboratorio de instrumental topográfico y el usuario haya seleccionado la práctica a realizar, se le ofrece el acceso al oportuno tutorial (vídeo didáctico en el mundo real, alojado en Canal_UPM Youtube (Benito Oterino, Martínez Peña, \& Chueca Castedo, 2013), imprescindible para poder realizar la práctica en TOPLAB), donde se muestran los elementos básicos del instrumental, accesorios, procedimiento de observación, detalle de datos a registrar, comprobaciones que deben hacerse en campo y cálculos que conlleva cada observación hasta lograr los oportunos resultados finales, tal como deberá hacerlo el alumno cuando realice la observación real en campo. Además de estos video-tutoriales de las observaciones reales, está previsto producir vídeos con el propio laboratorio virtual para mostrar las peculiaridades de la observación virtual al interactuar con TOPLAB.

Seguidamente, TOPLAB muestra un menú que contiene la “Guía” (Fig. 5) con el detalle de operaciones que el alumno debe ir realizando para completar la práctica.

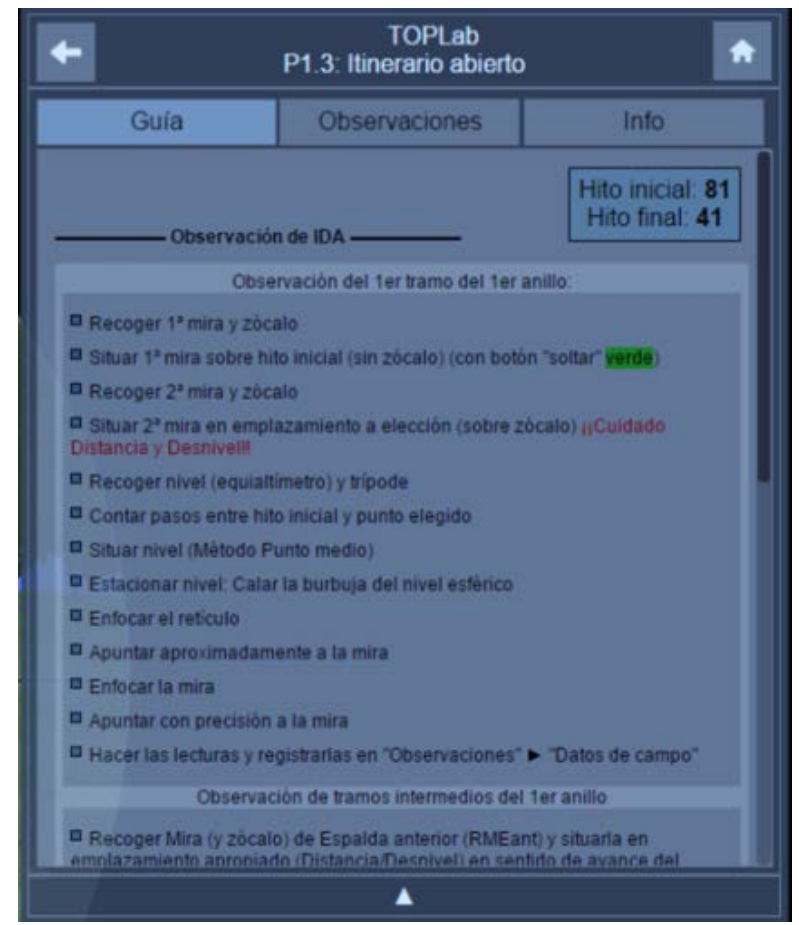

Fig. 5 Guía de operaciones a realizar para desarrollar la P1.3

El avatar, guiado por el alumno según la cadencia de la guía, ira recogiendo el instrumental y los accesorios necesarios, saldrá al “campo de prácticas” y los estacionará convenientemente en los puntos elegidos por él mismo o asignados por TOPLAB. En el momento de la colocación de cada mira, aparecerá el oportuno ayudante que la sustenta.

El usuario, a través de su avatar realizará las observaciones, interactuando con el instrumental, obtendrá los datos (lecturas de mira) y los introducirá en el Fichero de Observaciones (columnas correspondientes a "Datos de campo"), al que se accede desde el menú de la práctica.

Por último realizará los cálculos oportunos, los introducirá en las columnas de "resultados” y enviará el fichero para la verificación de la bondad de las comprobaciones de campo, la coherencia de sus observaciones y la revisión de los cálculos (Fig. 6). Gracias a la retro alimentación (ofrecida a través de código de colores sobre sus propios resultados) podrá repetir las observaciones o corregir los cálculos si fuera necesario.

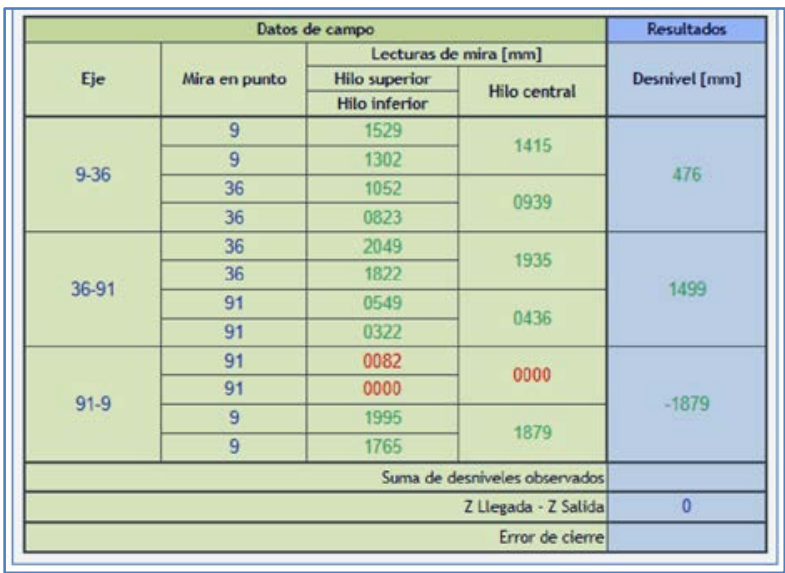

Fig. 6 Registro de los datos observados, cálculos realizados y resultados obtenidos

Cada práctica se considerará realizada cuando la verificación dé como resultado la aparición de todos los valores obtenidos "en verde".

El profesor accede a una Base de datos en red (Fig. 7) que se alimenta con los registros verificados por los alumnos.

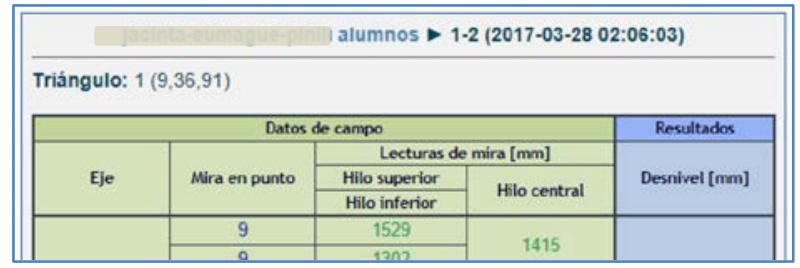

Fig. 7 BBDD en red

C. Descripción de los trabajos desarrollados para producir las prácticas de nivelación con TOPLAB (no se detallan los trabajos de programación realizados por los técnicos del GATE

Con el objetivo de contribuir a la producción de prácticas virtuales como recurso didáctico, se describen las fases que ha supuesto este proyecto.

El soporte técnico, imprescindible para la concreción en un laboratorio virtual de las ideas innovadoras del profesorado, queda restringido en la UPM a las iniciativas seleccionadas en los procesos competitivos convocados al efecto $y$ es proporcionado por el GATE. TOPLAB fue incluido en la resolución definitiva de laboratorios virtuales de 2 de febrero de 2015.

La solicitud de un laboratorio virtual implica desarrollar una idea inicial en cuanto a prácticas a implementar, instrumental a utilizar, procesos a desarrollar y resultados a obtener.

Una vez asegurado el soporte técnico, la primera fase del proyecto es la planificación inicial de los trabajos a desarrollar: reuniones entre el profesorado y el equipo de programación, visita de los técnicos al entorno real en el que se desarrollan las prácticas, captura de imágenes del instrumental a modelar y preparación de la documentación. 
Especial dedicación supone la creación y modelado del instrumental y los accesorios virtuales. También es necesario diseñar algunas partes del escenario como el edificio y el almacén de instrumental topográfico, así como el entorno (colinas, arboles...) incluyendo las señales que materializan los puntos con coordenadas conocidas (hitos, clavos, conos) que constituyen el marco de referencia que permite el control métrico de las observaciones virtuales.

La fase más compleja es la que supone determinar las adaptaciones al mundo virtual de las operaciones topográficas que se realizan en el mundo real. Requiere el trabajo coordinado de programadores y profesores de topografía para explotar al máximo los limitados recursos del visor en que se programa el laboratorio, en favor de hacer las prácticas virtuales lo más realistas, a la vez que lo más didácticas posible.

Es imprescindible una revisión continua de la interpretación que el equipo técnico hace de las directrices marcadas por el profesorado, lo que supone una permanente retroalimentación entre programadores y profesores. Para ello es determinante avanzar sobre versiones intermedias.

Esta revisión continua se aplica también a la definición de los criterios para valorar la bondad de las observaciones virtuales, así como la correcta ejecución de los cálculos realizados por los alumnos. Esta fase está apoyada por la creación de hojas Excel muy completas que no solo calculan con los datos tomados por los alumnos sino que, en aplicación del cálculo inverso, proporcionan también a TOPLAB las lecturas instrumentales que corresponden a cada toma de datos, en función de las posiciones ocupadas por el instrumental y los accesorios.

En el caso que se describe, se redactaron numerosos documentos para recoger y tratar adecuadamente, fallos puntuales, fallos de concepto o circunstancias que por su carácter suponían buscar alternativas a la situación del mundo real para poder contemplarlas en el laboratorio virtual.

\section{RESUltados}

A. Resultados de aprendizaje esperados en la nivelación virtual

La realización de las prácticas de nivelación permite al estudiante ampliar su conocimiento de los niveles practicando los procedimientos de observación y captura de datos de campo y comprobando los resultados obtenidos. El alumno, una vez desarrolladas las prácticas, debe ser capaz de:

- Conocer las operaciones que conlleva la puesta en estación de los niveles.

- Conocer el procedimiento de nivelación simple para la obtención del desnivel entre dos puntos mediante nivelación geométrica.

- Conocer los procedimientos para la observación de itinerarios de nivelación.

La fase de nivelación geométrica virtual con TOPLAB está disponible desde el pasado mes de abril. La planificación para su verificación y difusión es la siguiente:

- Mayo: Cuestionario a los alumnos del grado en Ingeniería Geomática y Topografía (asignatura “Topografía y Geodesia”).
- Mayo: Cuestionario a los alumnos del Certificado de Profesionalidad "Levantamientos y replanteos".

- Mayo, junio: Presentación a profesores de otras ETSI de la UPM y recogida de su impresión a través de cuestionario.

- Junio: Ofrecimiento en abierto a profesores y usuarios en general

- $\quad$ A partir de septiembre: Utilización en las ETSI de la UPM en asignaturas de topografía así como en otros centros y por otros usuarios

\section{B. Evaluación. Opinión del alumno}

Mediante una encuesta específica (Benito Oterino J. , 2017) para valorar la usabilidad de este recurso didáctico se ha evaluado la opinión de dos grupos de alumnos, con diferentes características, que han trabajado con el laboratorio virtual:

- Grupo “Grado” (Estudios universitarios): alumnos (9) de 20 años de edad media, que realizaron la observación virtual antes de hacer la real.

- Grupo “FP” (Formación profesional): alumnos (16) de 39 años de edad media que habían realizado tres prácticas de nivelación previamente a utilizar el laboratorio virtual.

La encuesta se ha elaborado siguiendo criterios de usabilidad, en términos de eficiencia, eficacia y satisfacción del alumno. En las figuras 8.1 y 8.2 se recogen los resultados (muy similares) para uno y otro grupo de alumnos en términos de la media obtenida para cada pregunta (1: "Totalmente en desacuerdo” y 4: “Totalmente de acuerdo”).

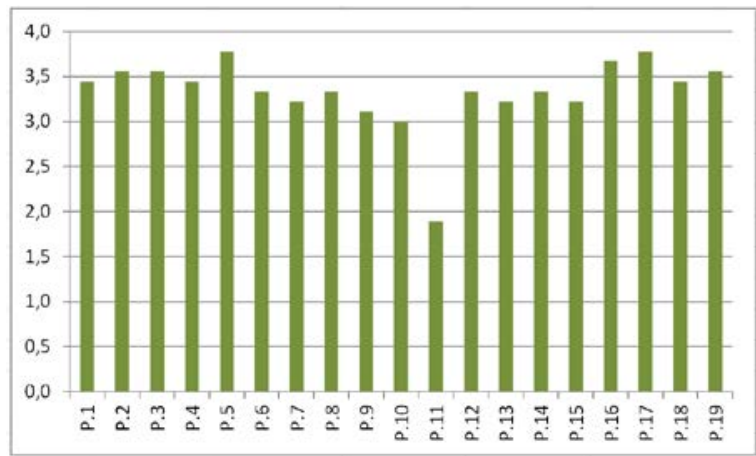

Fig. 8.1 Grupo “Grado”. Media obtenida por cada pregunta

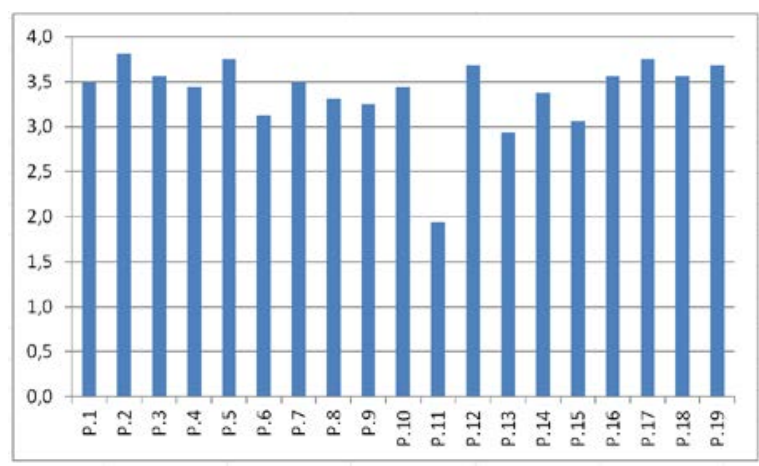

Fig. 8.2 Grupo "FP”. Media obtenida por cada pregunta

Las preguntas que obtienen un mayor grado de satisfacción son P5 y P17, coincidiendo ambas con 3.8 de media en los dos grupos de estudio. En el grupo FP, además obtiene este mismo grado de satisfacción la pregunta P2: 
- P 5: TOPLAB cumple el propósito para el que ha sido diseñado (complementar la docencia presencial)

- $\quad$ P 17: TOPLAB ofrece un contenido útil

- $\quad$ P 2: TOPLAB reproduce fielmente los niveles óptico y electrónico así como los accesorios (miras, trípodes, zócalos)

La media global de satisfacción alcanza 3.4 en el grupo "Grado" y 3.5 en el grupo "FP", exceptuando en ambos grupos la pregunta 11, única redactada en sentido desfavorable ("He necesitado realizar varias veces cada práctica en TOPLAB para asimilarla totalmente"), que obtiene una media de 1.9 en ambos grupos: casi la mitad de los alumnos asimilan la práctica realizándola una sola vez.

\section{Impacto en usuarios externos a la UPM.}

Se recogió también la opinión de 25 profesionales en ingeniería Geomática que participaron en el Taller experimental desarrollado en el XI Congreso Internacional de Geomática y Ciencias de la Tierra, TOPCART 2016, celebrado del 26 al 30 de octubre, donde profesores, comerciales y técnicos interactuaron con la versión pruebas de las prácticas de nivelación geométrica del laboratorio virtual y mostraron su satisfacción por la disponibilidad en abierto de este recurso didáctico que, mayoritariamente, calificaron de excelente.

\section{CONCLUSIONES}

La realidad virtual supone un recurso didáctico motivador que facilita el autoaprendizaje, muy apreciado por los alumnos, y que resulta de gran utilidad para emular prácticas, complementando las sesiones presenciales.

En el ámbito del aprendizaje autónomo en abierto en formación no reglada, ofrece posibilidades hasta ahora impensables por carestía del instrumental o alejamiento geográfico de los centros docentes.

La fiel modelización del instrumental y accesorios es importante para facilitar, después, su manejo en el mundo real.

La retroalimentación proporcionada a los alumnos y usuarios por el laboratorio virtual en el momento de la verificación de los datos, cálculos y resultados registrados es un complemento indispensable en el aprendizaje autónomo y es altamente valorado por los mismos.

Sin disponer aún de una muestra significativa, la impresión del profesorado al corregir las prácticas reales del presente curso es de mejores resultados que en cursos precedentes, atribuibles solo a haber tenido la experiencia previa del laboratorio virtual. El mayor aporte de esta experiencia, en el entorno de la clase invertida, está en que el alumno sale a sus prácticas de campo con entrenamiento previo proporcionado por el laboratorio virtual, tanto en el uso del instrumental como en el método de trabajo. En el campo del aprendizaje adaptativo, reafirma los conocimientos del alumno que, por sus características, necesite refuerzo tras la realización convencional de la práctica. En cualquier caso, potencia el trabajo autónomo del alumno en actividades dependientes de instrumental sólo disponible en el centro, en circunstancias muy concretas de horario, condiciones meteorológicas, etc.

Quizá la rigidez en la secuencia de acciones necesarias para completar una observación pueda considerarse como debilidad aunque esta forma de proceder responde, en gran medida, a los protocolos habituales de observación. Desde el punto de vista de creación del laboratorio, el tiempo y esfuerzo invertidos son muy altos aunque el rendimiento esperado compensará la inversión.

En cuanto a la realización técnica, es básica la retroalimentación continua de los dos equipos de trabajo, profesorado y programadores, presentando cada uno de ellos las acciones, variantes y posibilidades ante la operatividad que el visor ofrece $\mathrm{y}$ las adaptaciones que los procesos topográficos admiten en su paso al mundo virtual.

Las prácticas de nivelación geométrica en TOPLAB son totalmente sostenibles, pues están alojadas en la plataforma 3dlabs.upm, y son perfectamente transferibles a otros contextos como analíticas de aprendizaje (en proceso según proyecto de innovación educativa UPM TOPLAB_AA, convocatoria 2016) y MOOC de Observaciones Topográficas (en un próximo futuro).

en

\section{AGRADECIMIENTOS}

Este proyecto está siendo posible gracias a la estrecha y eficaz colaboración del GATE UPM (dirección y técnicos) con los profesores. El trabajo de programación es encomiable para adaptarse a las peculiaridades de las observaciones topográficas, siendo especialmente relevante la reproducción del terreno con el marco de referencia que hace posible validar las observaciones de campo.

\section{REFERENCIAS}

Arranz Justel, J., \& Soler García, C. (2015). Métodos topográficos. Madrid: Universidad Politécnica de Madrid, Escuela Técnica Superior de Ingenieros en Topografía Geodesia y Cartografía.

Benito Oterino, J. (Junio de 2017). Google Drive. Formulario "Nivelación geométrica virtual con TOPLAB". Obtenido de https://goo.gl/forms/FADtRf4GKMxsZ4h22

Benito Oterino, J., Martínez Peña, M., \& Chueca Castedo, R. (2013). El vídeo didáctico, facilitador del aprendizaje/autoaprendizaje. CINAIC, (págs. 325-330). Madrid.

http://www.dmami.upm.es/dmami/documentos/liti/ACTA S_CINAIC_2013.pdf.

GATE UPM. (2017). 3dlabs.upm. Obtenido de Topografía. TOPLAB: https://3dlabs.upm.es/laboratorios.php\#lab29

Grupo de Innovación Educativa INNGEO. (2005). INNGEO. Obtenido http://www2.topografia.upm.es/grupos/inngeo

Márquez, I. (2010). II Congreso internacional comunicación 3.0. Universidad de Salamanca. La simulación como aprendizaje: educación y mundos virtuales, (pág. 11). Salamanca.

Paniagua, S. (2006). Aprender haciendo, formación basada en simuladores. TELOS. Cuadernos de comunicación e innovación. Fundación telefónica.

Ruiz Gutierrez, J. (2013). La simulación como instrumento de aprendizaje (Evaluación de herramientas y estrategias de aplicación en el aula). 